\title{
Adaptable Radiative Transfer Innovations for Submillimeter Telescopes (ARTIST)
}

\author{
Marco Padovani ${ }^{1}$ and Jes K. Jørgensen ${ }^{2}$ \\ on behalf of the ARTIST team: \\ Frank Bertoldi $^{3}$, Christian Brinch ${ }^{4}$, Pau Frau ${ }^{1}$, \\ Josep Miquel Girart ${ }^{1}$, Michiel Hogerheijde ${ }^{4}$, Attila Juhasz ${ }^{4}$, \\ Rolf Kuiper $^{3}$, Reinhold Schaaf ${ }^{3}$, Wouter H. T. Vlemmings ${ }^{3}$ \\ ${ }^{1}$ Institut de Ciències de l'Espai (CSIC-IEEC) \\ Universitat Autònoma de Barcelona, Spain \\ email: [padovani,girart,frau] @ice.cat \\ ${ }^{2}$ Centre for Star and Planet Formation \\ University of Copenhagen, Denmark \\ email: jes@snm.ku.dk \\ ${ }^{3}$ Argelander Insitute for Astronomy \\ University of Bonn, Germany \\ email: [wouter, bertoldi,rschaaf,kuiper] Castro.uni-bonn.de \\ ${ }^{4}$ Leiden Observatory \\ University of Leiden, the Netherlands \\ email: [michiel,brinch,juhasz] @strw.leidenuniv.nl
}

\begin{abstract}
Submillimeter observations are a key for answering many of the big questions in modern-day astrophysics, such as how stars and planets form, how galaxies evolve, and how material cycles through stars and the interstellar medium. With the upcoming large submillimeter facilities ALMA and Herschel a new window will open to study these questions. ARTIST is a project funded in context of the European ASTRONET program with the aim of developing a next generation model suite for comprehensive multi-dimensional radiative transfer calculations of the dust and line emission, as well as their polarization, to help interpret observations with these groundbreaking facilities.
\end{abstract}

Keywords. radiative transfer, methods: numerical, stars: formation, submillimeter, polarization

\section{Introduction}

The Atacama Large Millimeter Array is the largest ground based project in astronomy. It will be the world's most powerful instrument for millimeter and submillimeter astronomy, providing enormous improvements in sensitivity, resolution and imaging fidelity in these wavelength bands. The main focus of the early use of ALMA, compared to present-day facilities, will be its high angular resolution and sensitivity. For studies of star formation, for example, ALMA will zoom in to AU scales in circumstellar disks in nearby star forming regions and thereby address some of the key questions in disk formation. It will also open up new possibilities in the study of planet formation, resolving the effects of planets on the disks around young stars and thus providing direct observational constraints on planet formation models. At the same time, the Herschel Space Observatory is providing high spatial and spectral resolution observations at wavelengths unobservable from the ground, in particular, of lines of $\mathrm{H}_{2} \mathrm{O}$ and high excitation transitions of molecules tracing the dense warm gas, e.g., in the innermost regions of protostars or in shocks. 
The simultaneous observation of dust and of a multitude of spectral lines opens new horizons for the physical and chemical analysis of the objects, e.g., to determine the excitation conditions (density, temperature, radiation), the chemical abundances and chemical network, and through polarization measurements, the magnetic field. It will be necessary to take all these observational constraints into account for a realistic quantitative description. ALMA will offer a new chance to study magnetic fields: due to receiver constraints, full polarization calibration and imaging will be the norm rather than the exception, making it essential that a self-consistent polarization modeling tool is available to the ALMA users.

With the novelty of these observations it will be critical to have an efficient, flexible and state-of-the-art modeling package that can provide a direct link between the theoretical predictions and the quantitative constraints from the submillimeter observations. The new observational opportunities require a new generation of modeling tools that can model the full multi-dimensional structure of, e.g., a low-mass protostar, including its envelope, disk, outflow and magnetic field, and their time evolution. Current tools are inadequate for the modeling of such complex structures because of their speed and inaccessibility, while tools to model polarization are completely lacking. Both ALMA and Herschel will provide us with large samples of sources, observed homogeneously as part of large key and legacy programs. This makes it prudent to have easily accessible and efficient tools, which with high convergence speed incorporate all observational constraints, for large source samples, and in a systematic fashion. It is the aim of this program to provide such an innovative suite of model tools and test it with existing submillimeter data and with new data from ALMA and Herschel.

\section{Objectives}

The goal of this project is to deliver a next generation radiative transfer modeling package that provides a self-consistent model for the emission of a multi-dimensional source observed at submillimeter wavelengths. For this project we are specifically motivated, without any loss of more general applicability, by low-mass star formation. Our modeling package shall be able to provide a self-consistent modeling tool for the line and continuum as well as polarization emission from, e.g., a young stellar object, incorporating an infalling large-scale envelope, rotationally decoupled protoplanetary disk, outflow cavity, and magnetic field - with no restrictions of the intrinsic geometry. With such an innovative model tool it will be possible to provide quantitative constraints on the relation between the large-scale angular momentum in the core and the disk evolution, on the direct impact of the outflows and their launching close to the disk surface, and on the importance of the magnetic field. Two important issues need to be addressed in this particular example: $(i)$ young stellar objects are characterized by structure on a wide range of spatial scales and with complex geometries, but current radiative transfer tools are locked to fixed linear or logarithmic grids and can therefore model multi-dimensional source structures only with great computational time expense; (ii) while most current observations indicate that magnetic fields play an important role in various stages of the star formation process, their relation to the physical source structure is yet poorly constrained. ALMA observations will study the magnetic fields through resolved line and continuum polarization observations that can not be properly analyzed with current models. The modeling tool will also be useful for tackling scientific questions relating to ALMA observations of, e.g., evolved stars, planetary nebulae or extragalactic starbursts. Providing radiative transfer tools that take complex source structures into account will also be of great help to interpret Herschel observations, e.g., to understand the origin of 
$\mathrm{H}_{2} \mathrm{O}$ and high excitation $\mathrm{CO}$ lines in the interface between protostellar cores and outflows or jets. There is a high degree of coupling between different modeling aspects: e.g., to understand the polarization of a given molecule's emission it is necessary to understand the physical conditions and chemical network that leads to the molecule's formation and excitation. For complex source structures it is necessary to develop an approach to time-dependent multi-dimensional modeling, e.g., through libraries of theoretical model prescriptions that can readily be incorporated and expanded for comparison to the data.

\section{Tools}

The planned model suite will have the following three components: $(a)$ an innovative radiative transfer code using adaptive gridding that allows simulations of sources with arbitrary multi-dimensional and time-dependent structures ensuring a rapid convergence and thus allowing an exploration of parameters; $(b)$ unique tools for modeling the polarization of the line and dust emission, information that will come with standard ALMA observations; (c) a comprehensive Python-based interface connecting these packages, thus with direct link to, e.g., ALMA data reduction software (CASA). A schematic overview of the program is shown in Fig. 1.

(a) - 3D Line Radiative Transfer: LIME. ALMA's high resolution data will produce the need to model phenomena with non-symmetric structures, such as spiral-waves, proto-planet resonances in evolving circumstellar disks, close protostellar binaries etc. Conventional radiative transfer tools use simple linear or logarithmic spatial grids. To model complex source structures in higher dimensions thus requires increasingly finer grid scaling, which becomes very difficult to handle computationally. As an alternative, the SimpleX algorithm (Ritzerveld \& Icke 2006) uses a Poisson method to define a grid based on the density distribution. The cornerstone of ARTIST is the 3D line radiative transfer code, LIME (Brinch \& Hogerheijde 2010), which utilizes the SimpleX gridding algorithm in a $3 \mathrm{D}$ extension to the RATRAN radiative transfer code (Hogerheijde \& van der Tak 2000). LIME is currently being applied to for example modeling new $\mathrm{H}_{2} \mathrm{O}$ observations of protostars and disks from the Herschel Space Observatory. For these models the adaptive gridding method ensures rapid convergence, for lines from molecules such as $\mathrm{H}_{2} \mathrm{O}$ that are far from LTE excitation.

(b) - Dust and Line Polarization. Various theoretical studies predict that magnetic fields, turbulence or/and magneto-hydrodynamic waves may be the main agents controlling both the evolution of molecular clouds and the star-formation process (e.g., Bertoldi \& McKee 1992; Mac Low \& Klessen 2004; Mouschovias et al. 2006; van Loo et al. 2007). Unfortunately, the magnetic field is the least-known observable in star formation, due to the inherent difficulty to measure it with present telescopes (mainly through polarimetric observations of dust and molecular emission; e.g., Girart, Rao \& Marrone 2006). This situation will change dramatically with ALMA, which will provide such an improvement in sensitivity that polarization observations of dust and molecular lines can be done in many sources at a very good angular resolution. However, the interpretation of the polarized data is difficult and appropriate tools are needed to scientifically harvest the wealth of polarization data expected from ALMA. Note that the ALMA band-7 (275-373 GHz) receivers will require full polarization calibration and imaging even for projects with no primary interest in polarization. The ARTIST package consists of a set of modeling tools for polarization and magnetic fields in different molecular and dust environments (e.g., low- and high-mass protostars, envelopes around evolved stars). 


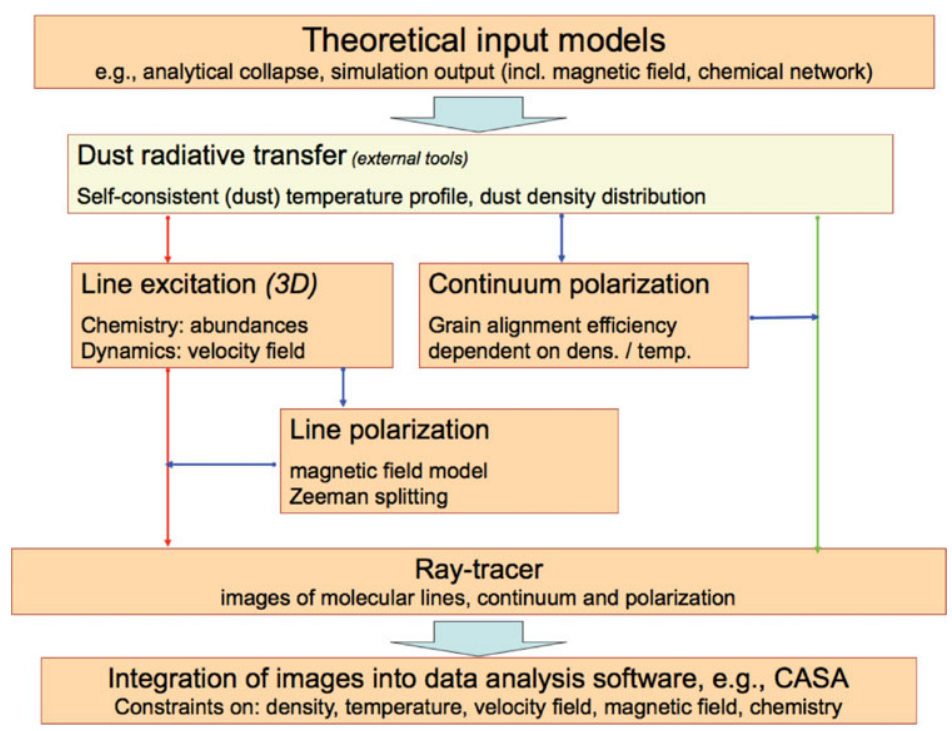

Figure 1. Schematic overview of the components in the ARTIST program.

(c) - Model interface and library. An important component of ARTIST is a common interface for the codes used for radiative transfer modeling of typical data produced by submillimeter telescopes such as ALMA and Herschel. The model interface will include: (i) a library of standard input models, e.g., for collapsing protostars, circumstellar disks and evolved stars; (ii) a wrapper package that links the existing dust and line radiative transfer codes; (iii) tools to analyze model output, e.g., to extract information about molecular excitation and its deviation from LTE, or optical depth surfaces, and to import this in existing visualization packages; $(i v)$ a ray tracing backend that readily provides data cubes that can be used in data reduction packages.

\section{Current Status}

The aim of the ARTIST project is to supply the community with the described tools in one coherent modeling package. The tools will be made publicly available as they are finished: we expect LIME to be released medio-2010 with the remaining components of the package to follow. The tools will be distributed and supported through the ALMA regional center nodes in Bonn and Leiden as well as the Danish initiative for Far-infrared and Submillimeter Astronomy (DFSA; Copenhagen, Denmark). For more information see http://www.astro.uni-bonn.de/ARC/artist.

\section{References}

Bertoldi, F. \& McKee, C. F. 1992, ApJ, 395, 140

Brinch, C. \& Hogerheijde, M. 2010, A\&A, in press (arXiv:1008.1492)

Girart, J. M., Rao, R. \& Marrone, D. P. 2006 Science, 313, 812

Hogerheijde, M. M. \& van der Tak, F. F. S. 2000, A\&SA, 362, 697

MacLow, M.-M. \& Klessen, R. S. 2004, RvMP, 76, 125

Mouschovias, T. Ch., Tassis, K. \& Kunz, M. W. 2006, ApJ, 646, 1043

Ritzerveld, J. \& Icke, V. 2006, PhRvE, 74, 26704

van Loo, S., Falle, S. A. E. G., Hartquist, T. W. \& Moore, T. J. T. 2007 A\&广A, 471, 213 